\title{
On the infimum attained by a reflected Lévy process
}

\author{
K. Dębicki · K.M. Kosiński • M. Mandjes
}

Received: 3 December 2010 / Revised: 31 March 2011 / Published online: 27 September 2011

(C) The Author(s) 2011. This article is published with open access at Springerlink.com

\begin{abstract}
This paper considers a Lévy-driven queue (i.e., a Lévy process reflected at 0 ), and focuses on the distribution of $M(t)$, that is, the minimal value attained in an interval of length $t$ (where it is assumed that the queue is in stationarity at the beginning of the interval). The first contribution is an explicit characterization of this distribution, in terms of Laplace transforms, for spectrally one-sided Lévy processes (i.e., either only positive jumps or only negative jumps). The second contribution concerns the asymptotics of $\mathbb{P}\left(M\left(T_{u}\right)>u\right)$ (for different classes of functions $T_{u}$ and $u$ large); here we have to distinguish between heavy-tailed and light-tailed scenarios.
\end{abstract}

Keywords Lévy processes · Fluctuation theory - Queues · Heavy tails · Large deviations

Mathematics Subject Classification (2000) Primary 60K25 · Secondary 60G51

\footnotetext{
K. Dębicki

Instytut Matematyczny, University of Wrocław, pl. Grunwaldzki 2/4, 50-384 Wrocław, Poland e-mail: Krzysztof.Debicki@math.uni.wroc.pl

K.M. Kosiński (凶) · M. Mandjes

Korteweg-de Vries Institute for Mathematics, University of Amsterdam, Amsterdam, The Netherlands

e-mail: K.M.Kosinski@uva.nl

M. Mandjes

e-mail: M.R.H.Mandjes@uva.nl

K.M. Kosiński · M. Mandjes

Eurandom, Eindhoven University of Technology, Eindhoven, The Netherlands

M. Mandjes

CWI, Amsterdam, The Netherlands
} 


\section{Introduction}

The class of processes with stationary and independent increments, known as Lévy processes, form a key object in applied probability. A substantial body of literature is devoted to Lévy processes that are reflected at 0 , sometimes also referred to as Lévydriven queues, and are regarded as a valuable generalization of the classical M/G/1 queues; also, the important special case of reflected Brownian motion is covered.

These reflected Lévy processes are defined as follows. Let $X \equiv\{X(t): t \in \mathbb{R}\}$ be a Lévy process with (without loss of generality) zero drift: $\mathbb{E} X(1)=0$ and $X(0)=0$. Then define the queueing process (or: workload process, storage process) $Q \equiv\{Q(t): t \geq 0\}$ through (for $c>0$ )

$$
Q(t):=\sup _{s \leq t}(X(t)-X(s)-c(t-s)),
$$

where it is assumed that the workload is in equilibrium (stationarity) at time 0 , i.e., $Q(0)={ }_{\mathrm{d}} Q_{e}$.

We refer to this process $Q$ as the reflection of the Lévy process $Y=\{Y(t): t \in \mathbb{R}\}$ at 0 , where $Y(t):=X(t)-c t$. In the sequel we normalize time such that $c=1$.

When considering the steady state $Q_{e}$ of the reflected process introduced above, the literature can be roughly divided into two categories. (A) In the first place there are results on the full distribution of $Q_{e}$, in terms of the corresponding Laplace transform. Particularly for the case of one-sided jumps, these transforms are fairly explicit. If $X$ is such that it has only positive jumps and is not a subordinator (an increasing process), $X \in \delta^{+}$(which is often referred to as the spectrally positive case), then a generalization of the classical Pollaczek-Khintchine formula was derived [22], while in the case of only negative jumps, $X \in 8^{-}$(spectrally negative), $Q_{e}$ was seen to be exponentially distributed. In the Lévy processes literature $[6,17]$, this type of results can be found under the denominator fluctuation theory. We recall that there are powerful tools available for numerical inversion of Laplace transforms $[1,13]$. (B) In the second place there are results that describe the asymptotics of $\mathbb{P}\left(Q_{e}>u\right)$ for $u$ large. Then one has to distinguish between results in which the upper tail of the Lévy increments is light on the one hand, sometimes referred to as the Cramér case, and results that correspond to the heavy-tailed regime on the other hand; see for instance [16] and references therein.

In the present short communication, we consider a related problem: we analyze how long the process consecutively spends above a given level. More formally, we consider the distribution of $M(t):=\inf _{s \in[0, t]} Q(s)$, i.e., the minimum value attained by the workload process in a window of length $t$, where it is assumed that the queue is in stationarity at the beginning of the interval. This problem has various applications: one could for instance think of the analysis of persistent overload in an element of a communication network or a node in a supply chain; see e.g. [18]. A related study on the situation of infinitely-divisible self-similar input is [2].

Our results correspond to both branches (A) and (B) mentioned above: in Sect. 2 we present results on the Laplace transform of $M(t)$, relying on known results for Lévy fluctuation theory; we also consider the special case of Brownian motion. Section 3 identifies the asymptotics of $\mathbb{P}\left(M\left(T_{u}\right)>u\right)$ for different classes of functions $T_{u}$ and $u$ large; as expected, we need to distinguish between heavy-tailed and light-tailed input. 
Recall that

$$
Y(t)=X(t)-t, \quad Q(t)=\sup _{s \leq t}(Y(t)-Y(s)), \quad M(t)=\inf _{s \in[0, t]} Q(s) ;
$$

we will also extensively use the following notation:

$$
K(t):=\inf _{s \in[0, t]} Y(s) .
$$

It is well known that the process $Q$ admits the following representation (see [20, p. 375]):

$$
Q(t)=Q(0)+Y(t)+\max (0,-Q(0)-K(t))
$$

so that, for any $u>0$,

$$
\mathbb{P}(M(t)>u)=\mathbb{P}(Q(0)+K(t)>u) .
$$

Notice that due to the independent increments property of $X$, the random variables $Q(0)$ and $K(t)$ are independent, and hence $Q(0)+K(t)={ }_{\mathrm{d}} Q_{e}+K(t)$.

\section{Transforms for the spectrally one-sided case}

In this section we evaluate the double transform, with $x \geq 0, y>0$,

$$
\mathcal{L}(x, y):=\int_{0}^{\infty} \int_{0}^{\infty} e^{-x u} e^{-y t} \mathrm{~d} \mathbb{P}(M(t) \leq u) \mathrm{d} t=\int_{0}^{\infty} \mathbb{E} e^{-x M(t)} e^{-y t} \mathrm{~d} t .
$$

As indicated in the introduction, we do so for Lévy processes with one-sided jumps. We separately treat the spectrally-positive and the spectrally-negative case.

It is worth noticing that the double transform $\mathcal{L}$ uniquely determines the distribution of $M(t)$. Determining the probability distributions from the double transform requires Laplace inversion. It is noted that recently, substantial progress has been made with respect to this type of inversion techniques. Besides the 'classical' reference [1], we wish to draw attention to significant recent progress in [13]; the latter reference specifically addresses the multidimensional transforms, and also provides a fairly complete literature overview.

Let us start with computations that are valid for any Lévy process $X$ as introduced in Sect. 1. Integration by parts yields

$$
\mathcal{L}(x, y)=\int_{0}^{\infty} e^{-y t}\left(1-x \int_{0}^{\infty} e^{-x u} \mathbb{P}(M(t)>u) \mathrm{d} u\right) \mathrm{d} t=\frac{1}{y}-x \mathcal{K}(x, y),
$$

where

$$
\begin{aligned}
\mathcal{K}(x, y) & :=\int_{0}^{\infty} \int_{0}^{\infty} e^{-x u} e^{-y t} \mathbb{P}(M(t)>u) \mathrm{d} u \mathrm{~d} t \\
& =\int_{0}^{\infty} \int_{0}^{\infty} e^{-x u} e^{-y t} \mathbb{P}(Q(0)+K(t)>u) \mathrm{d} u \mathrm{~d} t \\
& =\int_{0}^{\infty} \int_{0}^{\infty} e^{-x u} e^{-y t} \int_{u}^{\infty} \mathbb{P}(K(t)>u-z) \mathrm{d} \mathbb{P}(Q(0) \leq z) \mathrm{d} u \mathrm{~d} t,
\end{aligned}
$$


where the last equality follows from the independence of $Q(0)$ and $K(t)$. Our goal is to evaluate the 'double transforms' $\mathcal{K}(x, y)$ and $\mathcal{L}(x, y)$ that uniquely determine the distribution of $M(t)$.

Let $R_{z}:=\inf \{t \geq 0:-Y(t)>z\}$ denote the first passage time of $-Y$ over level $z>0$; note that $Y$ has a negative drift, and therefore $R_{z}$ is finite almost surely. As the event $\{K(t)>-z\}$ coincides with $\left\{R_{z}>t\right\}$, we obtain, after interchanging the order of integration,

$$
\mathcal{K}(x, y)=\int_{0}^{\infty} \int_{0}^{z} e^{-x u}\left(\int_{0}^{\infty} e^{-y t} \mathbb{P}\left(R_{z-u}>t\right) \mathrm{d} t\right) \mathrm{d} u \mathrm{~d} \mathbb{P}(Q(0) \leq z) .
$$

\subsection{Spectrally-positive case}

Theorem 1 Let $X \in s^{+}$. Denote $\vartheta(s)=\log \mathbb{E} e^{-s Y(1)}$. Then with $x \geq 0, y>0$,

$$
\mathcal{L}(x, y)=\frac{1}{y} \frac{x \vartheta^{\prime}(0)}{\vartheta(x)} \frac{\vartheta^{-1}(y)}{y} \frac{\vartheta(x)-y}{x-\vartheta^{-1}(y)}
$$

Proof It is well known that $\mathbb{E} e^{-y R_{z}}=e^{-z \vartheta^{-1}(y)}$. Noting that

$$
\int_{0}^{\infty} e^{-y t} \mathbb{P}\left(R_{z-u}>t\right) \mathrm{d} t=\frac{1}{y}\left(1-\mathbb{E} e^{-y R_{z-u}}\right)=\frac{1}{y}\left(1-e^{-(z-u) \vartheta^{-1}(y)}\right),
$$

it follows that

$$
\mathcal{K}(x, y)=\int_{0}^{\infty}\left(\left(\frac{1-e^{-x z}}{x y}\right)-\left(\frac{e^{-z \vartheta^{-1}(y)}-e^{-x z}}{\left(x-\vartheta^{-1}(y)\right) y}\right)\right) \mathrm{d} \mathbb{P}(Q(0) \leq z) .
$$

Recalling that $Q(0)={ }_{\mathrm{d}} Q_{e}$ and using 'Pollaczek-Khintchine' we know that $\mathbb{E} e^{-\alpha Q_{e}}=$ $\alpha \vartheta^{\prime}(0) / \vartheta(\alpha)$, for any $\alpha>0$. Therefore the claim follows from (1) and the fact that

$$
\mathcal{K}(x, y)=\frac{1}{x y}\left(1-\frac{x \vartheta^{\prime}(0)}{\vartheta(x)}\right)-\frac{\vartheta^{\prime}(0)}{\left(x-\vartheta^{-1}(y)\right) y}\left(\frac{\vartheta^{-1}(y)}{y}-\frac{x}{\vartheta(x)}\right)
$$

\subsection{Spectrally-negative case}

Theorem 2 Let $X \in s^{-}$. Denote by $\eta^{-1}(x)=\sup \{s \geq 0: \eta(s)=x\}$ the right-inverse of $\eta(s)=\log \mathbb{E} e^{s Y(1)}$. Then with $x \geq 0, y>0$,

$$
\mathcal{L}(x, y)=\frac{1}{y} \frac{\eta^{-1}(0)}{\eta^{-1}(0)+x} \frac{\eta^{-1}(y)+x}{\eta^{-1}(y)}
$$

Proof Notice that in this case, $Q(0)={ }_{\mathrm{d}} Q_{e}$ is exponentially distributed with parameter $\alpha:=\eta^{-1}(0)>0$. Thus,

$$
\begin{aligned}
\mathcal{K}(x, y) & =\int_{0}^{\infty} \int_{0}^{z} e^{-x u} \cdot \frac{1}{y}\left(1-\mathbb{E} e^{-y R_{z-u}}\right) \alpha e^{-\alpha z} \mathrm{~d} u \mathrm{~d} z \\
& =\frac{1}{(\alpha+x) y}\left(1-\int_{0}^{\infty} \mathbb{E} e^{-y R_{z}} \alpha e^{-\alpha z} \mathrm{~d} z\right) .
\end{aligned}
$$


The second factorization identity [17, p. 176] states that

$$
\int_{0}^{\infty} \mathbb{E} e^{-y R_{z}} e^{-\alpha z} \mathrm{~d} z=\frac{\kappa(y, \alpha)-\kappa(y, 0)}{\alpha \kappa(y, \alpha)},
$$

where in this spectrally-negative case, $\kappa(y, x)=\frac{y-\eta(x)}{\eta^{-1}(y)-x}$. Now the claim follows from (1) and the fact that

$$
\mathcal{K}(x, y)=\frac{1}{(\alpha+x) y} \frac{\kappa(y, 0)}{\kappa(y, \alpha)} .
$$

\subsection{Brownian motion}

Theorem 3 Let $X$ be a standard Brownian motion $B \equiv\{B(t): t \in \mathbb{R}\}$. Then, for each $t>0$,

$$
\mathbb{P}(M(t)>u)=\exp (-2 u)\left(2(1+t) \Psi(\sqrt{t})-\sqrt{\frac{2 t}{\pi}} \exp \left(-\frac{t}{2}\right)\right),
$$

where $\Psi(x)=\mathbb{P}(\mathcal{N}>x)$ for a standard normal random variable $\mathcal{N}$.

Proof Since $B \in 8^{-}, Q(0)={ }_{\mathrm{d}} Q_{e}$ has an exponential distribution with mean $1 / 2$. Thus,

$$
\begin{aligned}
\mathbb{P}(M(t)>u) & =\mathbb{P}\left(Q(0)+\inf _{s \in[0, t]}(B(s)-s)>u\right) \\
& =\int_{u}^{\infty} \mathbb{P}\left(\inf _{s \in[0, t]}(B(s)-s)>u-x\right) 2 \exp (-2 x) \mathrm{d} x \\
& =2 \exp (-2 u) \int_{0}^{\infty} \mathbb{P}\left(\sup _{s \in[0, t]}(B(s)+s)<y\right) \exp (-2 y) \mathrm{d} y \\
& =\exp (-2 u) \mathbb{E} \exp \left(-2 \sup _{s \in[0, t]}(B(s)+s)\right)
\end{aligned}
$$

and the claim follows after some elementary computations (see also [7, (1.1.3)] or [5]).

\section{Asymptotics}

In this section we consider the asymptotics of $\mathbb{P}\left(M\left(T_{u}\right)>u\right)$ for a variety of functions $T_{u}$ and $u$ large. As usual, heavy-tailed and light-tailed scenarios need to be addressed separately.

\subsection{Heavy-tailed case}

In this section we shall work with the following assumption about the Lévy process $X$ : 
Assumption 1 For $\alpha>1$, let $X(1) \in \mathcal{R} \mathcal{V}(-\alpha)$-the class of distributions with a complementary distribution function that is regularly varying at $\infty$ with index $-\alpha$. Moreover, if $\alpha \in(1,2)$, then in addition

$$
\lim _{x \rightarrow \infty} \frac{\mathbb{P}(X<-x)}{\mathbb{P}(X>x)}=\rho \in[0, \infty) .
$$

We start with the following general proposition.

Proposition 1 Let $X$ be a Lévy process such that $\mathbb{E} X(1)=0$. Then, for any $\varepsilon>0$,

$$
\lim _{u \rightarrow \infty} \mathbb{P}\left(\left|\frac{K(u)}{u}+1\right|>\varepsilon\right)=0 .
$$

Proof First note that for such $X$ we have

$$
\frac{X(t)}{t} \rightarrow 0 \quad \text { a.s. or equivalently } \frac{Y(t)}{t} \rightarrow-1 \text { a.s. }
$$

Fix $\varepsilon>0$, then

$$
\begin{aligned}
\mathbb{P}\left(\left|\frac{K(u)}{u}+1\right|>\varepsilon\right) & =\mathbb{P}\left(\frac{1}{u} \inf _{t \in[0, u]} Y(t)>-1+\varepsilon\right)+\mathbb{P}\left(\frac{1}{u} \inf _{t \in[0, u]} Y(t)<-1-\varepsilon\right) \\
& =: I_{1}(u)+I_{2}(u) .
\end{aligned}
$$

Now (2) implies that

$$
I_{1}(u) \leq \mathbb{P}\left(\frac{Y(u)}{u}>-1+\varepsilon\right) \rightarrow 0 .
$$

Observe that, for any $T \leq u$,

$$
\begin{aligned}
I_{2}(u) \leq & \mathbb{P}\left(\frac{1}{u} \inf _{t \in[0, u]} Y(t)<-1-\varepsilon, \inf _{t \in[0, T]} Y(t)<\inf _{t \in[T, u]} Y(t)\right) \\
& +\mathbb{P}\left(\frac{1}{u} \inf _{t \in[0, u]} Y(t)<-1-\varepsilon, \inf _{t \in[0, T]} Y(t) \geq \inf _{t \in[T, u]} Y(t)\right) \\
\leq & \mathbb{P}\left(\inf _{t \in[0, T]} Y(t)<\inf _{t \in[T, u]} Y(t)\right)+\mathbb{P}\left(\frac{1}{u} \inf _{t \in[T, u]} Y(t)<-1-\varepsilon\right) \\
= & : I_{21}(u)+I_{22}(u) .
\end{aligned}
$$

Again (2) implies that $I_{21}(u) \rightarrow 0$. As for $I_{22}(u)$, note that

$$
I_{22}(u) \leq \mathbb{P}\left(\frac{1}{u} \inf _{t \in[T, u]} X(t)<-\varepsilon\right) .
$$

Now we will show that for any $\delta>0$ and $u$ large enough we have

$$
\mathbb{P}\left(\frac{1}{u} \inf _{t \in[T, u]} X(t)<-\varepsilon\right)<\delta,
$$


which proves that $I_{22}(u) \rightarrow 0$ and completes the proof.

Indeed, by (2), $T$ can be chosen such that

$$
\mathbb{P}(\forall t \geq T:|X(t)|<\delta t)>1-\delta .
$$

Therefore, for such $T$ and $\delta<\varepsilon$ we have

$$
\begin{aligned}
\mathbb{P}\left(\frac{1}{u} \inf _{t \in[T, u]} X(t)<-\varepsilon\right)= & \mathbb{P}\left(\frac{1}{u} \inf _{t \in[T, u]} X(t)<-\varepsilon, \forall t \geq T:|X(t)| \geq \delta t\right) \\
& +\mathbb{P}\left(\frac{1}{u} \inf _{t \in[T, u]} X(t)<-\varepsilon, \forall t \geq T:|X(t)|<\delta t\right) \\
< & \delta,
\end{aligned}
$$

where we used the fact that the last probability equals zero.

In the sequel we say that $f(n) \sim g(n)$ if $f(n) / g(n) \rightarrow 1$ as $n \rightarrow \infty$.

Proposition 2 Assume that the Lévy process X satisfies Assumption 1.

(i) If $f(n) \geq n$, then

$$
\mathbb{P}(X(n)>f(n)) \sim n \mathbb{P}(X(1)>f(n)),
$$

as $n \rightarrow \infty, n \in \mathbb{N}$.

(ii) As $u \rightarrow \infty$,

$$
\mathbb{P}\left(Q_{e}>u\right) \sim \frac{u}{\alpha-1} \mathbb{P}(X(1)>u) .
$$

Proof The asymptotics in (i) can be found in, e.g., [10] for $\alpha \geq 2$ and [8, 9] for $\alpha \in(1,2)$; see also [14] for a recent treatment. For (ii), see, for example, [3, 16].

We now state the main result of this subsection: the exact asymptotics of $\mathbb{P}\left(M\left(T_{u}\right)>u\right)$.

Theorem 4 Assume that the Lévy process $X$ satisfies Assumption 1. Then

$$
\mathbb{P}\left(M\left(T_{u}\right)>u\right) \sim \mathbb{P}\left(Q_{e}>u+T_{u}\right)+T_{u} \mathbb{P}\left(X(1)>u+T_{u}\right), \quad \text { as } u \rightarrow \infty .
$$

The asymptotics in Theorem 4 can be made more explicit. Part (ii) of Proposition 2 immediately leads to the following corollary.

Corollary 1 Assume that the Lévy process X satisfies Assumption 1. Then

$$
\mathbb{P}\left(M\left(T_{u}\right)>u\right) \sim \begin{cases}\frac{1}{\alpha-1} u \mathbb{P}(X(1)>u) & \text { when } T_{u}=o(u), \\ \frac{A+\alpha}{\alpha-1}(A+1)^{-\alpha} T_{u} \mathbb{P}\left(X(1)>T_{u}\right) & \text { when } u \sim A T_{u}, \\ \frac{\alpha}{\alpha-1} T_{u} \mathbb{P}\left(X(1)>T_{u}\right) & \text { when } u=o\left(T_{u}\right),\end{cases}
$$

as $u \rightarrow \infty$. 
Proof of Theorem 4 The proof consists of an upper bound and a lower bound. We use the notation $T_{u}^{-}:=\left\lfloor T_{u}\right\rfloor$ and $T_{u}^{+}:=\left\lceil T_{u}\right\rceil$.

Upper bound To prove an (asymptotically) tight upper bound for $\mathbb{P}\left(M\left(T_{u}\right)>u\right)$, first we observe that for any $\varepsilon>0$, using that $Q(0)={ }_{\mathrm{d}} Q_{e}$ is independent of $\{X(t)$ : $t \geq 0\}$,

$$
\begin{aligned}
\mathbb{P}\left(M\left(T_{u}\right)>u\right) \leq & \mathbb{P}\left(M\left(T_{u}^{-}\right)>u\right) \leq \mathbb{P}\left(Q_{e}+X\left(T_{u}^{-}\right) \geq u+T_{u}^{-}\right) \\
\leq & \mathbb{P}\left(Q_{e}>(1-\varepsilon)\left(u+T_{u}^{-}\right)\right)+\mathbb{P}\left(X\left(T_{u}^{-}\right)>(1-\varepsilon)\left(u+T_{u}^{-}\right)\right) \\
& \quad+\mathbb{P}\left(Q_{e}>\varepsilon\left(u+T_{u}^{-}\right)\right) \mathbb{P}\left(X\left(T_{u}^{-}\right)>\varepsilon\left(u+T_{u}^{-}\right)\right) \\
= & : \pi_{1}^{+}(u)+\pi_{2}^{+}(u)+\pi_{3}^{+}(u) .
\end{aligned}
$$

Using (i) of Proposition 2 and the strong law of large numbers for $X$, it is easy to show that $\pi_{3}^{+}(u)=o\left(\pi_{1}^{+}(u)\right)$ for a fixed $\varepsilon$. It is standard now to show that

$$
\lim _{\varepsilon \rightarrow 0} \limsup _{u \rightarrow \infty} \frac{\pi_{1}^{+}(u)}{\mathbb{P}\left(Q_{e}>u+T_{u}\right)}=1 .
$$

Moreover,

$$
\lim _{\varepsilon \rightarrow 0} \limsup _{u \rightarrow \infty} \frac{\pi_{2}^{+}(u)}{T_{u} \mathbb{P}\left(X(1)>u+T_{u}\right)}=1,
$$

due to item (i) in Proposition 2. This establishes the upper bound.

Lower bound As for the lower bound observe that

$$
\begin{aligned}
\mathbb{P} & \left(M\left(T_{u}\right)>u\right) \\
& \geq \mathbb{P}\left(M\left(T_{u}^{+}\right)>u\right) \geq \mathbb{P}\left(Q_{e}+K\left(T_{u}^{+}\right)>u, X\left(T_{u}^{+}\right)-T_{u}^{+}-K\left(T_{u}^{+}\right)<\varepsilon T_{u}^{+}\right) \\
& \geq \mathbb{P}\left(Q_{e}+X\left(T_{u}^{+}\right)>u+(1+\varepsilon) T_{u}^{+}\right) \mathbb{P}\left(X\left(T_{u}^{+}\right)-T_{u}^{+}-K\left(T_{u}^{+}\right)<\varepsilon T_{u}^{+}\right) \\
& =: \pi_{1}^{-}(u) \pi_{2}^{-}(u) .
\end{aligned}
$$

By Proposition $1, \pi_{2}^{-}(u) \rightarrow 1$ as $u \rightarrow \infty$. Also,

$$
\begin{aligned}
\pi_{1}^{-}(u) \geq & \mathbb{P}\left(Q_{e}+X\left(T_{u}^{+}\right)-\varepsilon T_{u}^{+} / 2>u+(1+\varepsilon / 2) T_{u}^{+}, X\left(T_{u}^{+}\right)>-\varepsilon T_{u}^{+} / 2\right) \\
\geq & \mathbb{P}\left(\left\{Q_{e}>u+(1+\varepsilon / 2) T_{u}^{+}, X\left(T_{u}^{+}\right)>-\varepsilon T_{u}^{+} / 2\right\}\right. \\
& \left.\cup\left\{X\left(T_{u}^{+}\right)>u+(1+\varepsilon / 2) T_{u}^{+}\right\}\right) \\
= & \mathbb{P}\left(Q_{e}>u+(1+\varepsilon / 2) T_{u}^{+}\right) \mathbb{P}\left(X\left(T_{u}^{+}\right)>-\varepsilon T_{u}^{+} / 2\right) \\
& \left.+\mathbb{P}\left(X\left(T_{u}^{+}\right)\right)>u+(1+\varepsilon / 2) T_{u}^{+}\right) \\
& \quad-\mathbb{P}\left(Q_{e}>u+(1+\varepsilon / 2) T_{u}^{+}\right) \mathbb{P}\left(X\left(T_{u}^{+}\right)>u+(1+\varepsilon / 2) T_{u}^{+}\right) \\
= & : \pi_{3}^{-}(u) \pi_{4}^{-}(u)+\pi_{5}^{-}(u)-\pi_{6}^{-}(u) \pi_{7}^{-}(u),
\end{aligned}
$$


where we again used that $Q(0)={ }_{\mathrm{d}} Q_{e}$ and $\{X(t): t \geq 0\}$ are independent. By the strong law of large numbers, $\pi_{4}^{-}(u) \rightarrow 1$ as $u \rightarrow \infty$. Moreover, it is easy to show that $\pi_{6}^{-}(u) \pi_{7}^{-}(u)=o\left(\pi_{3}^{-}(u)\right)$. Now the lower bound follows by noting that

$$
\lim _{\varepsilon \downarrow 0} \liminf _{u \rightarrow \infty} \frac{\pi_{3}^{-}(u)}{\mathbb{P}\left(Q_{e}>u+T_{u}\right)}=1
$$

and that (i) of Proposition 2 yields

$$
\lim _{\varepsilon \downarrow 0} \liminf _{u \rightarrow \infty} \frac{\pi_{5}^{-}(u)}{T_{u} \mathbb{P}\left(X(1)>u+T_{u}\right)}=1 .
$$

This completes the proof.

\subsubsection{Stable Lévy processes}

Following the notation from [21], let $S_{\alpha}(\sigma, \beta, \mu)$ be a stable law with index $\alpha \in(0,2)$, scale parameter $\sigma>0$, skewness parameter $\beta \in[-1,1]$ and drift $\mu \in \mathbb{R}$. We call $X$ an $(\alpha, \beta)$-stable Lévy process if $X$ is a Levy process and $X(1)$ has the same distribution as $S_{\alpha}(1, \beta, 0)$.

Let

$$
B(\alpha, \beta):=\frac{\Gamma(1+\alpha)}{\pi} \sqrt{1+\beta^{2} \tan ^{2}\left(\frac{\pi \alpha}{2}\right)} \sin \left(\frac{\pi \alpha}{2}+\arctan \left(\beta \tan \left(\frac{\pi \alpha}{2}\right)\right)\right),
$$

and let $X$ be an $(\alpha, \beta)$-stable Lévy process with $\alpha \in(1,2)$ and $\beta \in(-1,1]$. Then,

$$
\mathbb{P}(X(1)>u) \sim \frac{B(\alpha, \beta)}{\alpha} u^{-\alpha},
$$

see, for example, [19, Proposition 2.1]. Now Theorem 4 can be rephrased as follows.

Corollary 2 For an $(\alpha, \beta)$-stable Lévy process $X$ with $\alpha \in(1,2)$ and $\beta \in(-1,1]$,

$$
\mathbb{P}\left(M\left(T_{u}\right)>u\right) \sim \begin{cases}\frac{1}{\alpha-1} \frac{B(\alpha, \beta)}{\alpha} u^{1-\alpha} & \text { when } T_{u}=o(u), \\ \frac{A+\alpha}{\alpha-1}(A+1)^{-\alpha} \frac{B(\alpha, \beta)}{\alpha} T_{u}^{1-\alpha} & \text { when } u \sim A T_{u}, \\ \frac{\alpha}{\alpha-1} \frac{B(\alpha, \beta)}{\alpha} T_{u}^{1-\alpha} & \text { when } u=o\left(T_{u}\right),\end{cases}
$$

as $u \rightarrow \infty$.

\subsection{Light-tailed case}

In this subsection we consider the light-tailed situation, also frequently referred to as the Cramér case. Throughout, with $\phi(\vartheta):=\log \mathbb{E} \exp (\vartheta X(1))$ denoting the cumulant function, we impose the following assumption. 


\section{Assumption 2 Let}

$$
\beta^{\star}:=\sup \left\{\beta: \mathbb{E} e^{\beta X(1)}<\infty\right\} .
$$

Assume that $\beta^{\star}>0$ and there exists $\vartheta^{\star} \in\left(0, \beta^{\star}\right)$ such that $\phi\left(\vartheta^{\star}\right)=\vartheta^{\star}$.

For $r \geq 0$, define

$$
I(r):=\sup _{\vartheta>0}(\vartheta r-\phi(\vartheta))
$$

Proposition 3 Under Assumption 2, the following statements hold.

(i) As $u \rightarrow \infty$,

$$
\log \mathbb{P}\left(Q_{e}>u\right) \sim-\vartheta^{\star} u
$$

(ii) For all $u>0$,

$$
\mathbb{P}\left(Q_{e}>u\right) \leq e^{-\vartheta^{\star} u} .
$$

(iii) The function I obeys

$$
I(1)<\infty \quad \text { and } \quad I^{\prime}(1) \leq \vartheta^{\star} .
$$

(iv) For any $\varepsilon>0$,

$$
\liminf _{u \rightarrow \infty} \frac{1}{u} \log \mathbb{P}(K(u)>-\varepsilon u) \geq-I(1) .
$$

Proof For (i) and (ii), we refer to [4, Theorems 5.1 and 5.2 of Chap. XIII] or [15, Proposition 5.1 and Remark 5.1]. For (iii), notice that $I(1)=\sup _{\vartheta>0}(\vartheta-\psi(\vartheta))$ is attained for $\vartheta \in\left(0, \vartheta^{\star}\right)$; therefore also $I^{\prime}(1) \leq \vartheta^{\star}$. As for (iv), observe that

$$
\mathbb{P}(K(u)>-\varepsilon u)=\mathbb{P}\left(\frac{Y(u \cdot)}{u} \in A_{\varepsilon}\right)
$$

where

$$
A_{\varepsilon}:=\{f \in D[0,1]: f(t)>-\varepsilon, \forall t \in[0,1]\}
$$

and $D[0,1]$ is the space of càdlàg functions on $[0,1]$. Using sample-path large deviations results for Lévy processes (see [11, Theorems 5.1 and 5.2]), we now obtain that

$$
\liminf _{u \rightarrow \infty} \frac{1}{u} \log \mathbb{P}(K(u)>-\varepsilon u) \geq-\inf \left\{\psi(f): f \in A_{\varepsilon} \cap C[0,1]\right\},
$$

where $\psi(f):=\int_{0}^{1} I\left(f^{\prime}(t)+1\right) \mathrm{d} t$. Now observe that the path $f^{\star} \equiv 0$ is in $A_{\varepsilon}$. The stated follows by realizing that $\psi\left(f^{\star}\right)=I(1)$.

Now we can proceed with the main result of this subsection. 
Theorem 5 Assume that the Lévy process X satisfies Assumption 2. Then

$$
\log \mathbb{P}\left(M\left(T_{u}\right)>u\right) \sim-u \vartheta^{\star}-T_{u} I(1), \quad \text { as } u \rightarrow \infty .
$$

The asymptotics in Theorem 5 can trivially be made more explicit by comparing both exponential decay rates. The intuition behind the following corollary is that, in large deviations language, the most likely path corresponding to the rare event under study first builds up from an empty system to level $u$ (at time 0), and then remains at level $u$ for the nest $T_{u}$ time units; both parts of the path result in both contributions to the decay rate (i.e., $-u \vartheta^{\star}$ and $-T_{u} I(1)$ ). Then, depending on whether $T_{u}$ is small or large with respect to $u$, one of these two contributions dominates.

Corollary 3 Assume that the Lévy process $X$ satisfies Assumption 2. Then

$$
\mathbb{P}\left(M\left(T_{u}\right)>u\right) \sim \begin{cases}-u \vartheta^{\star} & \text { when } T_{u}=o(u), \\ -T_{u}\left(A \vartheta^{\star}+I(1)\right) & \text { when } u \sim A T_{u}, \\ -T_{u} I(1) & \text { when } u=o\left(T_{u}\right),\end{cases}
$$

as $u \rightarrow \infty$.

Proof of Theorem 5 The proof again consists of two bounds.

Lower bound Observe that the probability of interest is, for any $\varepsilon>0$, bounded from below by

$$
\mathbb{P}\left(Q_{e}>u+\varepsilon T_{u}\right) \mathbb{P}\left(K\left(T_{u}\right)>-\varepsilon T_{u}\right) .
$$

Now the lower bound follows by combining parts (i) and (iv) of Proposition 3, and then sending $\varepsilon \downarrow 0$.

Upper bound Observe that

$$
K(t) \leq(X(t)-t) \mathbb{I}(t)
$$

where $\mathbb{I}(t)$ denotes the indicator function $1_{\{X(t) / t \in(0,1)\}}$. Thus,

$$
\begin{aligned}
\mathbb{P}\left(M\left(T_{u}\right)>u\right) & \leq \mathbb{P}\left(Q_{e}+\left(X\left(T_{u}\right)-T_{u}\right) \mathbb{I}\left(T_{u}\right)>u\right) \\
& =\int_{\mathbb{R}} \mathbb{P}\left(Q_{e}>u-x T_{u}+\mathbb{I}\left(T_{u}\right)\right) \mathrm{d} \mathbb{P}\left(\frac{X\left(T_{u}\right)}{T_{u}} \mathbb{I}\left(T_{u}\right) \leq x\right) \\
& =\int_{0}^{1} \mathbb{P}\left(Q_{e}>u-x T_{u}+T_{u}\right) \mathrm{d} \mathbb{P}\left(\frac{X\left(T_{u}\right)}{T_{u}} \mathbb{I}\left(T_{u}\right) \leq x\right) \\
& \leq e^{-\vartheta^{\star} u} \int_{0}^{1} e^{-\vartheta^{\star} T_{u}(1-x)} \mathrm{d} \mathbb{P}\left(\frac{X\left(T_{u}\right)}{T_{u}} \mathbb{I}\left(T_{u}\right) \leq x\right),
\end{aligned}
$$

where the last inequality follows from part (ii) of Proposition 3. The sequence $\{X(u) \mathbb{I}(u) / u\}$ satisfies the large deviations principle on $((0,1), \mathcal{B}(0,1))$ with rate $u$ 
and rate function $I(\cdot)$. Thus, Varadhan's lemma [12, Theorem 4.3.1] implies

$$
\begin{aligned}
\lim _{u \rightarrow \infty} \frac{1}{T_{u}} \log \int_{0}^{1} e^{-\vartheta^{\star} T_{u}(1-x)} \mathrm{d} \mathbb{P}\left(\frac{X\left(T_{u}\right)}{T_{u}} \mathbb{I}\left(T_{u}\right) \leq x\right) & =-\inf _{x \in(0,1)}\left(\vartheta^{\star}(1-x)+I(x)\right) \\
& =I(1),
\end{aligned}
$$

where the last equality is due to part (iii) of Proposition 3 and convexity of $I(\cdot)$.

Acknowledgements K. Dębicki was supported by MNiSW Grant N N201 394137 (2009-2011) and by a travel grant from NWO (Mathematics Cluster STAR). K.M. Kosiński was supported by NWO Grant 613.000.701. K. Dębicki and M. Mandjes thank the Isaac Newton Institute, Cambridge, for hospitality.

Open Access This article is distributed under the terms of the Creative Commons Attribution Noncommercial License which permits any noncommercial use, distribution, and reproduction in any medium, provided the original author(s) and source are credited.

\section{References}

1. Abate, J., Whitt, W.: Numerical inversion of Laplace transforms of probability distributions. ORSA J. Comput. 7, 36-43 (1995)

2. Albin, J., Samorodnitsky, G.: On overload in a storage model, with a self-similar and infinitely divisible input. Ann. Appl. Probab. 14, 820-844 (2004)

3. Asmussen, S.: Subexponential asymptotics for stochastic processes: extremal behavior, stationary distributions and first passage probabilities. Ann. Appl. Probab. 8, 354-374 (1998)

4. Asmussen, S.: Applied Probability and Queues, 2nd edn. Springer, Berlin (2002)

5. Baxter, G., Donsker, M.: On the distribution of the supremum functional for processes with stationary independent increments. Trans. Am. Math. Soc. 85, 73-87 (1957)

6. Bertoin, J.: Lévy Processes. Cambridge University Press, Cambridge (1996)

7. Borodin, A., Salminen, P.: Handbook of Brownian Motion-Facts and Formulae. Birkhäuser, Basel (1996)

8. Borovkov, A.: Estimates for the distribution of sums and maxima of sums of random variables when the Cramér condition is not satisfied. Sib. Math. J. 41, 811-848 (2000)

9. Borovkov, A., Boxma, O.: On large deviation probabilities for random walks with heavy tails. Sib. Adv. Math. 13, 1-31 (2003)

10. Cline, D., Hsing, T.: Large deviation probabilities for sums of random variables with heavy or subexponential tails. Technical report, Texas A\& M University (1994)

11. De Acosta, A.: Large deviations for vector-valued Lévy processes. Stoch. Process. Appl. 51, 75-115 (1994)

12. Dembo, A., Zeitouni, O.: Large Deviations Techniques and Applications, 2nd edn. Springer, New York (1998)

13. Den Iseger, P.: Numerical transform inversion using Gaussian quadrature. Probab. Eng. Inf. Sci. 20, 1-44 (2006)

14. Denisov, D., Dieker, A., Shneer, V.: Large deviations for random walks under subexponentiality: the big-jump domain. Ann. Probab. 36, 1946-1991 (2008)

15. Dębicki, K., Es-Saghouani, A., Mandjes, M.: Transient asymptotics of Lévy-driven queues. J. Appl. Probab. 47, 109-129 (2010)

16. Dieker, A.: Applications of factorization embeddings for Lévy processes. Adv. Appl. Probab. 38, 768-791 (2006)

17. Kyprianou, A.: Introductory Lectures on Fluctuations of Lévy Process with Applications. Springer, Berlin (2006)

18. Pacheco, A., Ribeiro, H.: Consecutive customer losses in regular and oscillating $\mathrm{M}^{X} / \mathrm{G} / 1 / n$ systems. Queueing Syst. 58, 121-136 (2008) 
19. Port, S.: Stable processes with drift on the line. Trans. Am. Math. Soc. 313, 805-841 (1989)

20. Robert, P.: Stochastic Networks and Queues. Springer, Berlin (2003)

21. Samorodnitsky, G., Taqqu, M.: Stable Non-Gaussian Random Processes: Stochastic Models with Infinite Variance. Chapman \& Hall, London (1994)

22. Zolotarev, V.: The first passage time of a level and the behaviour at infinity for a class of processes with independent increments. Theory Probab. Appl. 9, 653-661 (1964) 